No new solutions

Nature's Price: The Economics of Mother Earth. By W. van Dieren and M.G.W. Hummelinck. Pp.193. (Marion Boyars: London and Boston, 1979.) £3.50.

THE authors of this book have tried to relate economics to ecology. They attempt to set a price on the natural assets of the Earth, the Sun and wind, the soil and water, on minerals and, particularly, on wildlife. Their thesis is that by underestimating the cost of these resources and the way they are wasted in the effort to achieve our so-called progress, we are entering into some very bad bargains. They try to shock us into reversing our attempts to secure unlimited industrial growth and ever-increasing agricultural productivity, which they say are leading rapidly to the destruction of the globe on which we live.

As a conservationist, and one who wishes to see the world's flora and fauna and our wild $\epsilon$ nvironment protected for the enjoyment of posterity, I hoped to find some new solutions to our problems, and to be taught new arguments to forward our aims. Unfortunately this book makes no striking new contributions to its subject, and it contains a good deal of ecological nonsense. The authors' aims are admirable, but they seldom hit the right target.

The reader has surely the right to expect that a book dealing with The Economics of Mother Earth contains accurate financial information, so that costs can be properly compared. Many pages are devoted to pest control, spelling out the dangers of chemical pesticides as against the advantages of non-chemical controls. Chemicals are shown (in my opinion rightly) to have potential ecological dangers, and to be expensive. The value of a pest-eating bird is more difficult to quantify. However, the figures for chemicals are seriously incorrect. We are told that "the world spent $£ 16,000$ million ( $\$ 30,000$ million) on chemical pesticides in 1973"'; in fact the world bill was only a tenth of this figure. Even in 1978, when chemical prices had risen threefold following the increase in oil prices, the sum involved was still only about half that suggested by van Dieren and Hummelinck. This error affects much of the discussion.

Elsewhere there are other misleading statements. In a discussion on the danger of producing strains of insects resistant to insecticides (a very real problem, I agree) we are told that "malaria is occurring on a greater scale than ever before. In Sri Lanka, the number of malaria victims has increased from a few hundred to two millions in the last fifteen years". This shocking increase has indeed taken place in Ceylon, but not because the vector cannot be controlled. This is an instance of the success of the extreme conservationists,

who caused the use of DDT to be stopped, so malaria once again became epidemic. Of course insecticide resistance is a real problem, as stressed in the recent report on Agricultural Pollution of the UK Royal Commission on Environmental Pollution, but in most countries where malaria control continues, the disease decreases and insecticides continue to be one of the important weapons ensuring this decrease.

The sections dealing with agriculture are equally disappointing. All the old misinformation about the inefficiency of modern mechanised farming is repeated. We have the dogmatic statement that "harvests cannot keep on growing by putting more money (artificial fertiliser, machines, pesticides) into the ground"', and we are assured repeatedly that a diverse population of wild animals will ensure better and more stable cropping. Would that these things were true; the life of the conservationist would then be easy. These authors can never have seen a patch of mealies grown in an area of the richest ecological diversity that has been visited by an elephant. Though bad arable farming can have disastrous results on the soil and

\section{Phenomena of superconductivity}

Magnetic Flux Structures in Superconductors. By R.P. Huebener. Pp.259. (Springer: Berlin, Heidelberg and New York, 1979.) DM59; \$32.50.

THIS book originates from the conference of the same title held at Argonne National Laboratory, 1973, and from subsequent graduate lecture courses. The presentation of material in the book assumes a familiarity with the phenomena of superconductivity although, perhaps reflecting its origins in a lecture course, results which are probably already well known, such as the quantisation of flux, are briefly derived again. The mathematics is everywhere very simple: the pattern throughout is to outline the physical ideas, derive the fundamental equation and then where appropriate to quote others' results, possibly even in simplified form. The emphasis is on exposing the reader to the basic physical phenomena and the diverse experimental techniques which are used to investigate them. Many references (over 500) are given throughout the text to enable those so inclined to follow up any of the topics.

The first half of the book is devoted to the static case. The different intermediate state structures of Type I supcrconductors are discussed and there are a number of photographs which illustrate the richness of the varieties observed in different materials and geometries. Discussion of the mixed state of Type II superconductors follows a brief introduction to the the environment in Britain and in the Netherlands (from which country this book originates) good intensive farming is steadily improving soil fertility; but most wildlife (except the soil flora and fauna, which flourishes) plays little part in the scenario.

This is all very sad. These authors have clearly the best of intentions. They recognise the very real dangers of overpopulation and of uncontrolled resource depletion, and make some not very original suggestions regarding their prevention. But once more they make us realise that, if we wish to preserve wildlife, we must come clean and give the right reasons, which are that we like to have it around, and that we should not pretend that they have such great economic value. In Britain we may regret the extinction of the bear and the wolf, but their reintroduction, though interesting to the enthusiast, would hardly increase the productivity of our farmers.

Kenneth Mellanby

Kenneth Mellanby was Director of Monks Wood Experimental Station from 1961-74.

Ginsburg-Landau theory. Interactions between one vortex line and another and with surfaces are calculated. The vortex lattices, defects and correlations with crystal lattices are described and illustrated. This section ends, with a review of nine techniques of investigating these flux structures.

The second half of the book looks at the dynamics of flux motion; because of the variety of effects and their complexity more use is made of referring the reader to original papers.

The motion of flux due to transport currents, changes in the magnetic field and temperature gradients are discussed phenomenologically; and the Hall effect, Ettinghausen and Peltier effects, the Josephson relation, transport entropy as well as instabilities and force free configurations are outlined. The time-dependent Ginsburg-Landau theory is carefully introduced and some results from it are given. The mechanisms for flux pinning has a short chapter as does flux creep and flux jumps. The techniques for studying the individual and collective motions of vortices are reviewed, and a chapter is devoted to electrical noise power analysis. The final chapter discusses the currentinduced resistive state with the emphasis on phenomena observed in thin films.

This book will be useful to researchers and to people involved in engineering aspects of superconductivity, particularly of small low field devices.

J.G.M. Armitage

J.G.M. Armitage is Lecturer in the Department of Physics at the University of St Andrews, UK 